\title{
HIRSCHSPRUNG'S DISEASE IN THE NEONATAL PERIOD*
}

\author{
BY \\ TH. EHRENPREIS \\ From the Karolinska Hospital, Stockholm
}

I do not intend to give an account of the changing philosophies concerning the aetiology and treatment of the disease which followed upon Hirschsprung's (1888) first presentation of it as an entity in 1886. It required about 60 years of vague or wrong ideas to find the key to our present knowledge. That happened when our attention was diverted from the characteristic megacolon and was focused on a less conspicuous and apparently normal distal part of the bowel.

In 1946 a study of the early stages of the disease in 10 newborn infants (Ehrenpreis, 1946) revealed that the megacolon was not present at birth but developed only secondarily. Hirschsprung's (1888) assumption that constipation was caused by congenital megacolon could thus be reversed: the megacolon proved to be caused by the congenital constipation.

During the next few years definite proof of the cause of congenital constipation was presented. Evidence was produced that a narrow or normallooking segment of rectosigmoid distal to the dilated colon was the primary pathology in Hirschsprung's disease (Swenson and Bill, 1948). Neurohistological investigations demonstrated that the entire distal segment lacked ganglion cells (Whitehouse and Kernohan, 1948; Bodian, Stephens and Ward, 1949; Swenson, Rheinlander and Diamond, 1949). Removal of the distal segment cured the disease (Swenson and Bill, 1948).

Finally, a most important advance was made by the subdivision of the group of idiopathic megacolon into two different disease entities (Bodian et al., 1949). Only about half of the cases of idiopathic megacolon showed the clinical, radiological and neurohistological signs which had been demonstrated as typical of Hirschsprung's disease. The other half disclosed a different pattern of symptoms and signs, the main diagnostic difference being the absence of a distal aganglionic segment (Figs. 1-2). This division has made it possible to account for the

\footnotetext{
* A paper given at the inaugural meeting of the British Association of Paediatric Surgeons in London in July, 1954.
}

controversial results of earlier investigations and, still more important, to select the cases suitable for surgical treatment by rectosigmoidectomy.

Ehrenpreis's (1946) study of the onset and early development of the disease in 10 newborn infants disclosed a disease pattern which is essentially different from the typical and well-known picture of chronic constipation, abdominal enlargement and radiological megacolon in later infancy and childhood.

\section{Illustrative Case}

In 1943 a 3-day-old infant was brought to the Crown Princess Louisa's Hospital in Stockholm with a tentative diagnosis of intestinal obstruction. Only a small amount of meconium had been passed. On the third day of lifethe baby started to vomit and the abdomen became increasingly distended. Clinical examination revealed $\bar{c}$ nothing abnormal except the abdominal distension.: A straight radiograph showed a moderate and diffuse gaseous distension of the small intestine and of the colon, so that any obstruction would be situated in the distal part of the colon. A barium enema, however, failed to reveal any obstruction, nor was any other abnormality demonstrable in the large bowel. The diagnosis was difficult. Clinically, the baby presented all the symptoms and signs of an intestinal obstruction. There seemed to be no reason why this otherwise healthy baby should develop a paralytic ileus. A diagnosis of Hirschsprung's disease was considered, and it was suggested that the typical dilatation of the colon might not necessarily be a congenital lesion but might have developed only as a result of congenital constipation. On reviewing the literature, however, no substantial support for such an assumption could be found, and the course of the case at first also seemed to refute the diagnosis. On evacuation of the barium enema, the bowels began to move, the abdominal distension subsided, and vomiting ceased. During one week of subsequent observation in the ward the baby appeared and behaved like an entirely normal and healthy infant. There were no feeding difficulties, no vomiting, no abdominal distension and normal bowel movements. At the age of 10 days the baby was discharged with a tentative diagnosis of meconium constipation. Not many days later, however, signs of intestinal obstruction again developed. At the age of 14 days the baby was readmitted with vomiting and abdominal distension. 


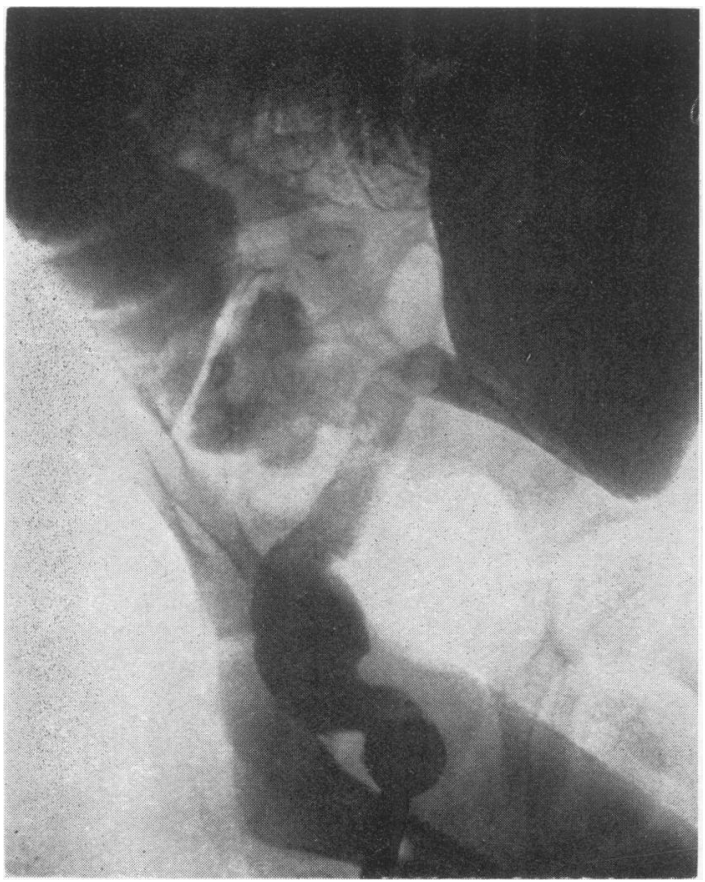

FIG. 1.-Narrow segment in Hirschsprung's disease.

No stools and no gas had been passed for 24 hours. A barium enema still failed to support a diagnosis of Hirschsprung's disease and evacuation of the enema again started bowel movements. After this, obstructive attacks reappeared with increasing frequency and intervals of apparent health successively came to be shorter and shorter. At the age of 5 weeks the classical disease picture of chronic constipation with abdominal enlargement developed, and the diagnosis of Hirschsprung's disease could also be confirmed radiologically.

This case was a very interesting experience. It exhibited a new picture of the disease, which in essential features diverged from the classical description. It now seemed desirable to find out if this picture was typical of the neonatal period or if it was only an exception.

The opportunity for further studies was soon offered. Three additional cases were admitted to the hospital within a short time and about a year later a fifth case appeared. From our files of Hirschsprung's disease five more cases could be traced back to the newborn period both clinically and radiologically. All of these 10 cases showed the same clinical pattern of repeated attacks of intestinal obstruction with intervals of apparent health and radiological absence of the typical megacolon during the neonatal period.

The three main obstructive symptoms of con- stipation, abdominal distension and vomiting have been tabulated according to their occurrence and day of onset in this series (Table 1). All of the

TABLE 1

CLINICAL ONSET OF MEGACOLON IN 10 NEWBORN INFANTS

\begin{tabular}{ll|c|c|c|c|c|c|c|c|c|c}
\hline \multicolumn{1}{c|}{ Case No. } & & 1 & 2 & 3 & 4 & 5 & 6 & 7 & 8 & 9 & 10 \\
\hline $\begin{array}{l}\text { Constipation } \\
\text { Distension }\end{array}$ &.. & 1 & 1 & 1 & 1 & 1 & 1 & 1 & $?$ & $?$ & 1 \\
Vomiting &. & 3 & - & 2 & 2 & 2 & $\frac{1}{2}$ & 5 & $2-3$ & 2 & 3 \\
\hline
\end{tabular}

The numbers define the age in days at appearance of the respective symptoms.

obstructive symptoms appeared fairly constantly during the first few days of life.

The radiological findings during the first week of life were evaluated on the basis of barium enema studies of 100 normal newborn infants. No significant dilatation of the colon was demonstrable at examination in the Hirschsprung series. As an example, three of the Hirschsprung colons have been photographed together with three normal colons (Fig. 3). I doubt if even the most experienced among my listeners would be able to distinguish between them.

In all the cases the obstructive symptoms subsided after the barium enema. The subsequent

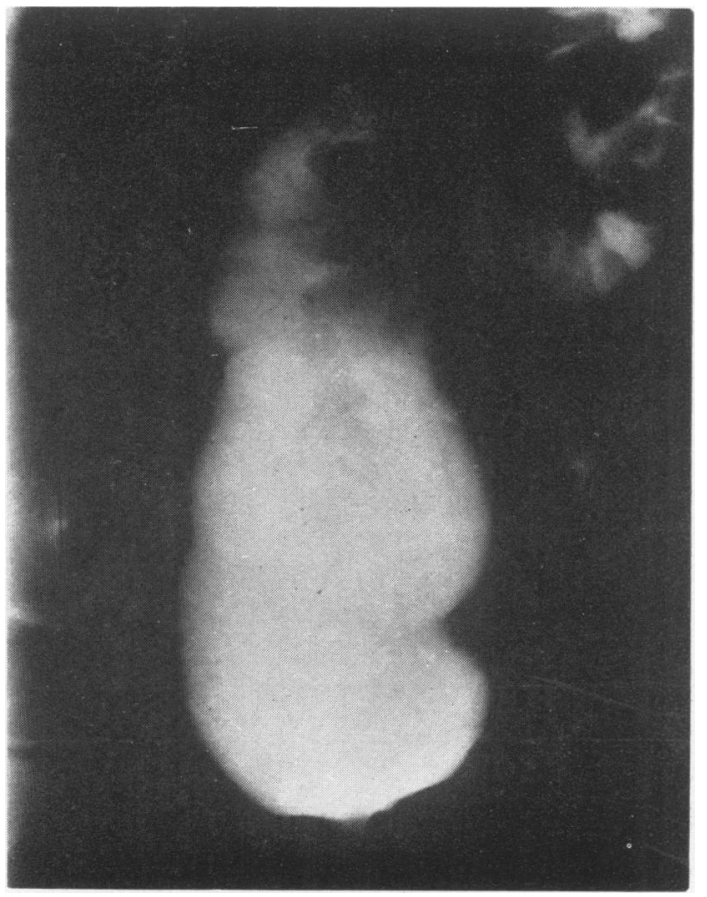

FIG. 2.-Rectal dilatation in chronic constipation. 


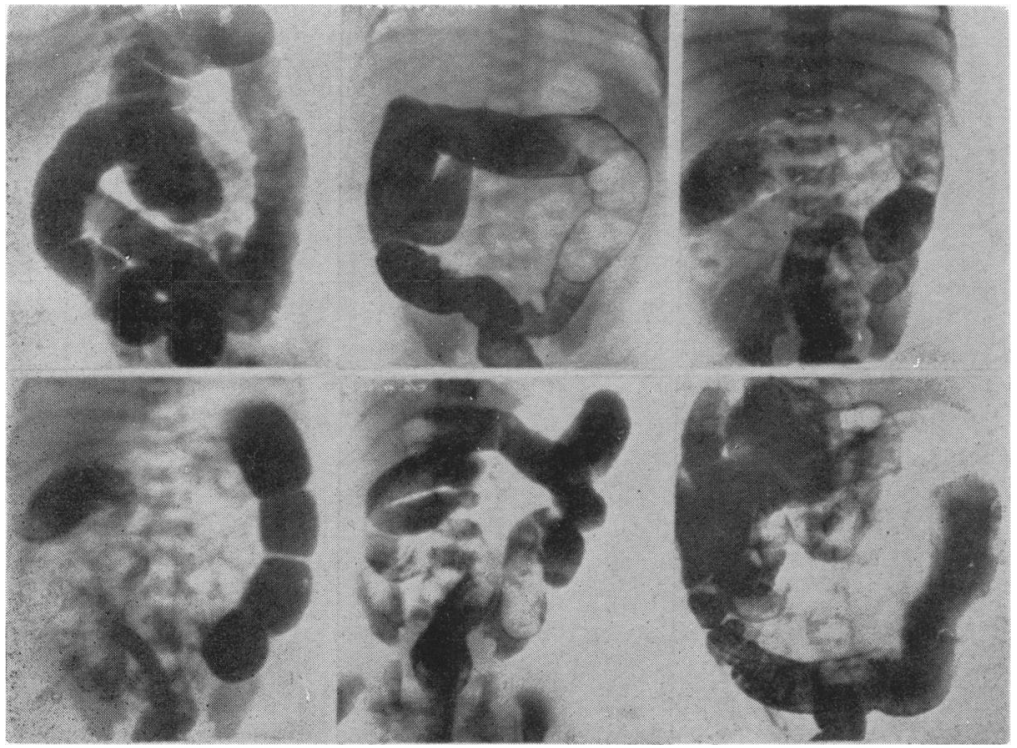

FiG. 3.-Radiographs of colon in three newborn infants with Hirschsprung's disease and three normal newborn infants.

course of the disease appears in Table 2 . In the majority of cases, a period of apparent health followed upon the relief of the initial obstruction. The length of this symptom-free period varied between one week and two and a half months in this series. Subsequently, obstructive periods alternated with intervals of apparent health for varying periods of time until the final transition into the classical morbid picture. The three main clinical characteristics of this picture, compared with the early stages, are chronic constipation, persistent abdominal enlargement, and absence of vomiting. As appears from Table 2, all these changes occurred more or less simultaneously in every single case, at ages varying between 3 or 4 weeks and 8 or 10 months.

Radiologically, all the cases developed the typical picture of megacolon. In four, barium enemas were repeated frequently enough to establish with a fair degree of accuracy the time required for the development of the radiological megacolon picture. The shortest time was 18 days and the longest time three and a half months (Fig. 4).

A synopsis of clinical and radiological data concerning the development of the disease picture suggests a pathophysiological explanation for the differences between the early and late stages. As long as the colon is of more or less normal size, the obstruction has an almost unlimited retrogressive effect, and the picture of acute intestinal obstruction results. Intervals of apparent health with normal bowel movements might be explained by reserve capacities of the still unaltered proximal colon, at least in cases with a short aganglionic segment. With the development of colon dilatation a kind of elastic reservoir is provided, which absorbs most of the retrogressive tendency. At this stage the proximal colon becomes more and more insufficient and finally loses power to over- 8 come the obstruction. Thus the picture of chronic constipation with abdominal enlargement results.

What still remains to be explained is the occurrence of free intervals in cases with a long aganglionic segment. I recently saw a case with an extremely long aganglionic segment, comprising the whole colon except the caecum. This child had developed a transitory intestinal obstruction during the first few days of life, which soon subsided. After this he appeared perfectly normal with daily bowel movements for about four months, when intestinal obstruction again developed and failed to respond to conservative treatment. A caecostomy was performed and biopsies were made from the sigmoid, transverse and proximal ascending colon. Microscopic examination revealed absence of ganglion cells from all three of these specimens. Since caecostomy the patient has thrived and is now

TABLE 2

DEVELOPMENT OF DEFINITE (CLASSICAL) CLINICAL PICTURE

\begin{tabular}{|c|c|c|c|c|c|c|c|c|c|c|c|}
\hline Case No. & & 1 & 2 & 3 & 4 & 5 & 6 & 7 & 8 & 9 & 10 \\
\hline Period of apparent health & following onset & $1 \mathrm{w}$. & $2 \mathrm{w}$. & $1 \mathrm{w}$. & - & $2 \frac{1}{2} \mathrm{~m}$ & - & $4 \mathrm{w}$. & - & $1 \mathrm{w}$. & $1 \mathrm{w}$. \\
\hline Beginning of persistent co & nstipation & - & $8 \mathrm{~m}$. & 3 w. & birth & $4 \frac{1}{2} \mathrm{~m}$ & birth & $4 \mathrm{~m}$. & birth & 4 w. & $3 \mathbf{w}$. \\
\hline Beginning of persistent & abdominal en- & $1 \mathrm{~m}$ & $8 \mathrm{~m}$ & 3. & ? & $<51 \mathrm{~m}$ & $>4 \mathrm{~m}$ & $<10 \mathrm{~m}$ & ? & $?$ & 一 \\
\hline Ceasing of vomiting & . $\quad \ldots$ & $1 \mathrm{~m}$. & $10 \mathrm{~m}$. & 5 w. & - & $6 \mathrm{~m}$ & $6 \mathrm{~m}$. & $8 \mathrm{~m}$. & $<1$ y. & $6 \mathrm{w}$. & $6 \mathrm{w}$. \\
\hline
\end{tabular}


awaiting colectomy with anastomosis between the lower rectum and the detached caecostomy or terminal ileum.* I have nothing to offer in the way of explanation for the long period of normal bowel movements in a case like this.

This specific disease pattern of the neonatal period and early infancy has been confirmed by several investigators (Bodian et al., 1949; Burnard, 1950; Swenson, 1951; Potts, Boggs and White, 1952).

\section{Diagnosis}

Before acquaintance with the early disease picture the diagnosis used to be something of a problem. Many cases were operated upon in the neonatal period with a diagnosis of intestinal obstruction. Usually operation was of no great help either as a diagnostic or a thera-

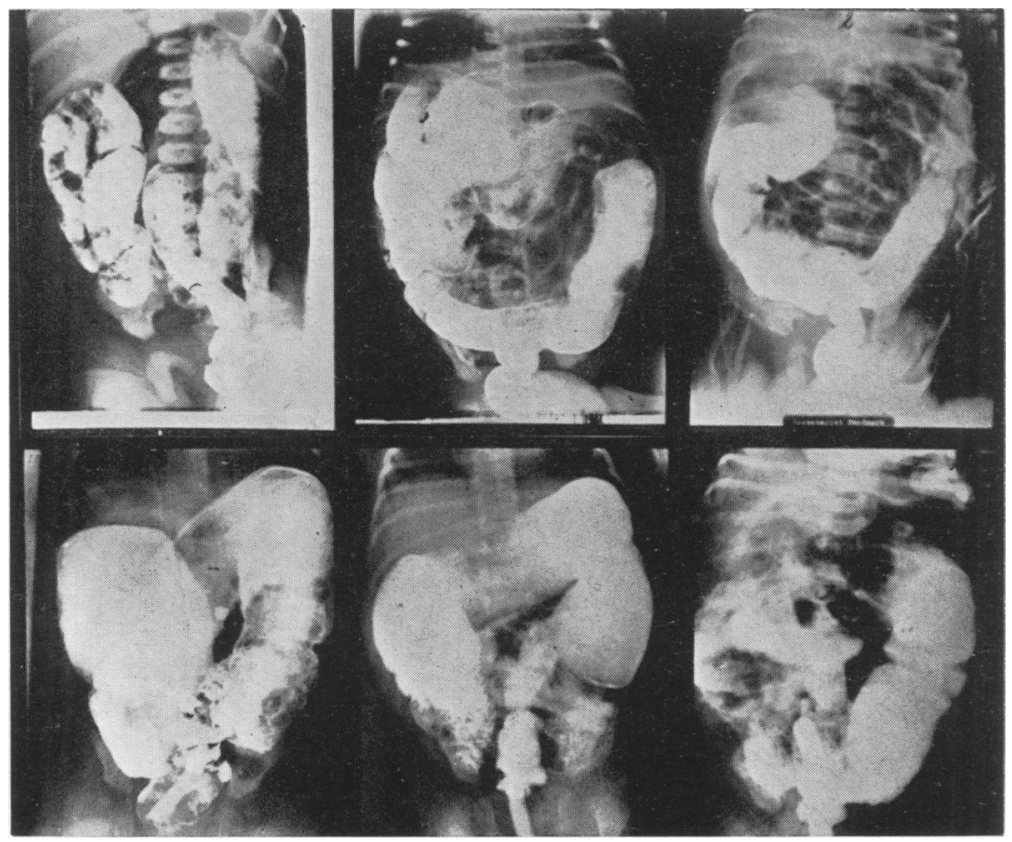

FIG. 4.-Radiological development of megacolon in three cases. Upper row: Newborn period. Lower row: Same cases at $3 \frac{1}{2}$ months, 5 weeks, and 6 weeks, respectively. peutic measure, as no cause

for the intestinal obstruction could be demonstrated. Now the diagnosis should offer no real difficulties. Every newborn baby presenting with signs of a low intestinal obstruction should be submitted to radiological examination. First the diagnosis of intestional obstruction has to be confirmed by plain films, showing distension of intestinal loops with fluid levels. Then, the cause for the obstruction has to be determined by barium enema studies. If a distal small but patent part of the colon (microcolon) fills, and if filling of the remainder of the colon proves impossible, there is probably an atresia in the colon. If the whole of the colon shows the characteristic microcolon (Fig. 5), there is a total obstruction above the colon. This obstruction might be due either to an atresia within the small bowel or to a meconium ileus. A more or less normallooking or slightly distended colon (Fig. 3) suggests an obstruction within the distal part of colon. There are two possible causes for such an obstruction. The more common cause is Hirschsprung's disease, more rarely simple meconium constipation. The immediate differentiation between these two conditions is often impossible, which, however, is of no great practical importance. In either event the

* Since this paper was submitted for publication a total colectomy with anastomosis between the terminal ileum and lower rectum has been successfully performed in this case. obstructive symptoms usually subside after a barium enema. Recurrence of obstructive symptoms suggests the diagnosis of Hirschsprung's disease, and repeated barium enemas will soon confirm this diagnosis (Fig. 4).

These simple diagnostic considerations were adopted about 10 years ago in Stockholm, and they have proved to be very helpful. Admittedly, there are other rare causes for a low intestinal obstruction in the newborn. Provided that due attention is paid to the clinical picture as a whole as well as to the radiological findings, the danger of missing a diagnosis of intestinal obstruction requiring surgery is slight.

The outlook for a newborn baby with Hirschsprung's disease used to be extremely poor. Out of the 10 patients discussed above, five died, four of them before the age of $1 \frac{1}{2}$ years. Similar and even higher mortality rates are given in other comparable series (Burnard, 1950; Potts et al., 1952; Hirschsprung, 1904; Ask-Upmark, 1930). With our present concepts of the aetiology, diagnosis and treatment of Hirschsprung's disease a definite change to a better prognosis has been accomplished for all age groups, even though the neonatal period is still a critical one. Larger series of newborn babies treated according to modern principles are still 


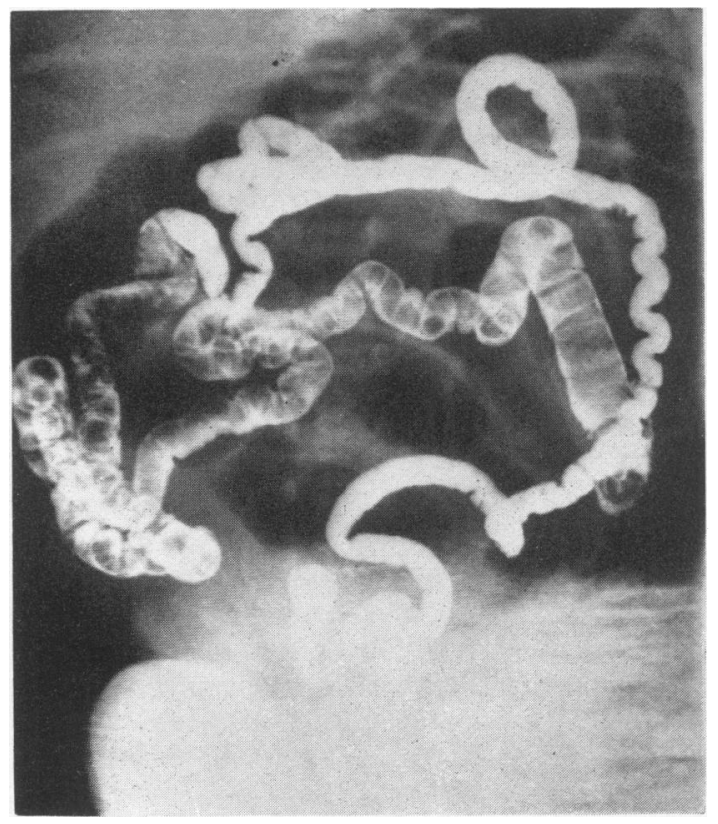

FIG. 5.-Microcolon and terminal ileum.

lacking, so I cannot give any exact figures as to present mortality rates of the initial stages of the disease.

\section{Treatment}

Present opinions as to the principles of treatment are practically unanimous. Resection of the aganglionic distal segment with anastomosis between the proximal dilated bowel and the lower rectum is the only treatment which eradicates the cause of the disease. This operation has been generally adopted as the treatment of choice and should be performed in every case with a definite diagnosis of Hirschsprung's disease.

When dealing specifically with the early stages of the disease the following considerations have to be taken into account. As long as the disease presents as attacks of recurring intestinal obstruction with intervals of apparent health there is generally no need for any kind of treatment, except occasional enemas or colonic irrigations. During this stage delimitation of the distal narrow segment is usually impossible or uncertain, either by radiological or surgical exploration. Should an obstructive attack fail to respond to conservative measures an emergency arises, which has to be dealt with by colostomy decompression of the proximal bowel. During an obstructive attack delimitation of the aganglionic segment might be possible, but resection in this stage would still not be the proper procedure because of the ileus. Resection is thus as a rule not practicable in early infancy.

As soon as the disease has entered into the fully developed stage, operation should be performed, usually as a one-stage procedure. Only if abdominal distension is not relieved by colonic irrigations, or when the patient's general condition does not seem to warrant the major procedure of rectosigmoidectomy, a preliminary colostomy has to be performed. The results of this operation have been most gratifying. Up to now reports of about 300 cases treated by rectosigmoidectomy have been published (Swenson, 1954; Bodian, 1952; Grob, 1952; Hallenbeck and Waugh, 1952; Ehrenpreis, 1952; Hiatt, 1951). The overall mortality rate is about $5 \%$. Except for some few failures due to incomplete resections, all of the patients have been relieved of their symptoms. Some of the patients have been followed now for six years, and so far, no recurrences have been reported after radical resection. Post-operative complications have been fews and transitory.

These excellent results testify to the physiological soundness of the present concept of Hirschsprung's disease and mark the rectosigmoidectomy operation as the first and only rational treatment.

\section{REFERENCES}

Ask-Upmark, E. (1930). Bruns' Beitr. klin. Chir., 151, 73.

Bodian, M. (1952). Practitioner, 169, 517.

Stephens, F. D. and Ward, B C. H (1949). Lancet, 1, 6 Burnard, E. D. (1950). Brit. med. J., 1, 151. Ehrenpreis, T. (1946). Acta chir. scand., 94, Suppl. 112 - (1952). Ibid., 102, 251.

Grob, M. (1952). Helv. chir. Acta, 19, 336.

Hallenbeck, G. A. and Waugh, J. M. (1952). Surg. Clin. N. Amer., 32, 1203.

Hiatt, R. B. (1951). Ann. Surg, 133, 321.

Hiatt, R. B. (1951). Ann. Surg., 133, 321.
Hirschsprung, H. (1888). Jb. Kinderheilk, 27, 1.

(1904). In Grancher, J. and Comby, J. Traite des maladies de l'enfance, 2 nd ed., vol. 2 , p. 241. Paris.

Potts, W. J., Boggs, J. D. and White, H. (1952). Pediatrics, 10, 253. Swenson, O. (1951). Ibid., 8, 542.

- (1954). J. Amer. med. Ass., 154, 651.

(1954). J. Amer. med. Ass., 154, 651.

and Bill, A. H. (1948). Surgery, 24, 212 . Rheinlander, $\mathrm{H}$.
Med., 241,551 .

Whitehouse, F. R. and Kernohan, J. W. (1948). Arch. intern. Med., 82, 75 . 